
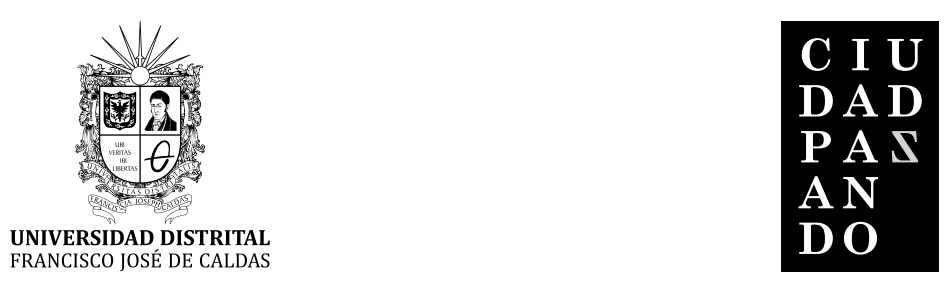

DOSSIER

Artículo de revisión

\title{
Las migraciones y la profundización de la crisis socioambiental
}

Migrations and the deepening of the socio-environmental crisis

Migrações e o aprofundamento da crise socioambiental

\section{César Augusto Ruiz Agudelo'}

Para citar: Ruiz, C. (2018). Las migraciones y la profundización de la crisis socioambiental. Revista Ciudad Paz-ando, 11(1), 25-31. doi: https://doi.org/10.14483/2422278X.12686

Fecha de recepción: 2 de noviembre de 2017

Fecha de aprobación: 13 de abril de 2018

1 Programa de Doctorado en Ciencias Ambientales y Sostenibilidad. Universidad Jorge Tadeo Lozano, Colombia. Correo electrónico: cesara ruiza@utadeo.edu.co 


\section{RESUMEN}

Las migraciones humanas son fenómenos masivos que configuran crisis humanitarias de escala global, las cuales, en una mirada transversal, van más allá de una cuestión de derechos laborales, civiles o sociales. En el presente artículo se revisa este fenómeno desde los tópicos de las migraciones humanas como causa y consecuencia de las crisis políticas y sociales, y las migraciones como causa y consecuencia de las crisis ambientales; lo anterior con referencia a que este fenómeno puede profundizar la crisis socioambiental global, que hoy en día es percibida con mayor intensidad. Controlar y gestionar los flujos migratorios es un reto transversal e involucra el reconocimiento de los valores y limitaciones ambientales.

Palabras clave: migraciones, migrantes ambientales, desplazados ambientales, crisis ambiental global.

\section{ABSTRACT}

Migrations are massive phenomena that figure humanitarian crises on a global scale, which, in a transversal perspective, go beyond the matter of labor, civil or social rights. In this article, this phenomenon is reviewed from the following perspectives: 1) - Migrations as a cause and consequences of political and social crises. 2) - Migrations as a cause and consequences of environmental crises. The aforementioned related to the fact that this phenomenon can deepen the global socio-environmental crisis, which nowadays is perceived with greater intensity. Controlling and managing migratory flows is a "transversal" challenge that involves the recognition of our environmental values.

Keywords: migrations, environmental migrants, environmental displacement, global environmental crisis.
As migrações são fenômenos maciços que moldam as crises humanitárias em escala global, que, em uma perspectiva transversal, vão além de uma questão de direitos trabalhistas, civis ou sociais. No presente artigo, este fenômeno é revisado das seguintes perspectivas: 1) - Migrações como causa e conseqüência de crises políticas e sociais. 2) - Migrações como causa e conseqüência de crises ambientais. $\mathrm{O}$ referido com referência ao fato de que esse fenômeno pode aprofundar a crise socioambiental global, que hoje em dia é percebida com maior intensidade. Controlar e gerenciar fluxos migratórios é um desafio "transversal" que envolve o reconhecimento de nossos valores ambientais.

Palavras-chave: migrações, migrantes ambientais, deslocados ambientais, crise ambiental global. 


\section{Introducción}

Holland, Peterson y González (2009), exponen con claridad que la inequidad en los ingresos es una variable determinante para la predicción de la pérdida de biodiversidad, en términos del aumento de las especies en categorías de amenaza o extinción; además de demostrar este aspecto econométricamente con datos panel de corte transversal en una muestra de países desarrollados y en vías de desarrollo, también encontraron que la densidad poblacional era otra variable que, para algunos países (en especial los desarrollados o más densamente poblados como India), es determinante en términos de aumentar la probabilidad de pérdida de biodiversidad, esto generado por la dependencia directa que expresan las poblaciones vulnerables sobre los recursos naturales derivados de la biodiversidad.

Se proyecta que para el 2050 serán más de 9000 millones de habitantes en el planeta tierra, lo que platea una potencial crisis socioambiental y de competencia por los recursos naturales cada vez más escasos (Rockström et al., 2009); en este contexto, es posible concluir que la biodiversidad (ese tejido vivo de la tierra que es la base de todos los procesos socioeconómicos) está en crisis, y que los países mega diversos (como Colombia, México y Brasil) están aumentando sus tasas de crecimiento poblacionales por fenómenos migratorios (Alemán, 2017). Es apenas lógico, siguiendo también a Holland et al. (2009), que el fenómeno migratorio sea una nueva preocupación para la gestión y conservación de la biodiversidad y los servicios ecosistémicos que sustentan a buena parte de la población del planeta.

Las migraciones son un fenómeno tan antiguo como la humanidad misma (Palacios, 2017), la única diferencia es que en la actualidad se han acentuado de forma dramática, poniendo en riesgo la integridad de los migrantes que se movilizan no solo en busca de mejores condiciones económicas, mejores condiciones ambientales o mejores condiciones de educación, sino por su propia supervivencia. Las migraciones humanas son fenómenos masivos que configuran crisis humanitarias de escala global, y desde una mirada verdaderamente transversal van más allá de una cuestión de derechos laborales, civiles o sociales.

En el presente artículo de reflexión se revisa este fenómeno desde los tópicos de las migraciones humanas como causa y consecuencia de las crisis políticas y sociales, y las migraciones humanas como causa y consecuencia de las crisis ambientales, "Los migrantes ambientales"; lo anterior con referencia a que este fenómeno puede llegar a profundizar la crisis socioambiental global, que hoy en día se percibe con mayor frecuencia e intensidad.

\section{Las migraciones humanas como causa y consecuencia de las crisis políticas y sociales}

Zaviršek (2017) define muy bien esta situación al expresar en su análisis que las personas pertenecientes a las migraciones masivas de hoy son la encarnación de una interrelación planetaria cada vez más perjudicial entre altas desigualdades, hegemonías y vidas humanas en permanente riesgo. De acuerdo con el autor, la sociedad está presenciando no solo una crisis humanitaria, sino una crisis fundamental del humanitarismo.

Siguiendo a Bradol (2004) cuando se habla de "crisis humanitaria" con referencia a los migrantes, se está afirmando que un gran número de personas están muriendo o se encuentran en riesgo de morir, que se enfrentan a la hambruna, la enfermedad, el agotamiento y circunstancias caóticas que ponen en riesgo total su existencia.

Un millón de personas que vivieron la guerra en Siria durante el 2015, con los niños que fueron enviados sin acompañamiento, hicieron lo que las personas atrapadas en medio de una crisis humanitaria hacen: buscaron escapar de la muerte. Solamente en septiembre de 2016, 170 bombas golpearon Aleppo, incluyendo dos bombas del cloro. Los dos hospitales más grandes fueron también bombardeados. En medio de esta situación caótica, la gente tomó una decisión razonable, responsable y valiente, demostrando su deseo de supervivencia y esperanza; sin embargo, una vez en su camino hacia la seguridad, fueron percibidos por la mayoría de los gobiernos, y también por las personas en los países en ruta, como bárbaros, violentos, mal intencionados y peligrosos para la salud pública. En 2015, por ejemplo, el presidente húngaro Victor Orban afirmó en repetidas ocasiones que todos los migrantes son una "amenaza a la seguridad", y elevó su tono en 2016 diciendo que son "venenosos" (Primer Ministro húngaro, 2016).

Los migrantes, sin embargo, en contra de cualquier expectativa razonable, salen de una crisis humanitaria solo para entrar en el grueso de una nueva (Zaviršek, 2017). No se puede aplicar el mismo tratamiento a 65,3 millones de desplazados forzosos al final de la década de los cincuenta, versus treinta y cinco millones de turistas que visitaron España en 2015 (Bernik, 2016). El viaje desde Siria o Turquía a Grecia cuesta entre 3000 y 5000 dólares por refugiado si todo sale bien, pero también puede costar vidas. Desde el 2000, alrededor de 30000 personas han muerto en el Mar Mediterráneo (Zaviršek, 2017).

Como se ha vuelto común en los discursos políticos modernos, la crisis humanitaria de los migrantes (en todo el planeta) se refleja en las fronteras estatales cada vez más militarizadas, buscando externalizar a la gente migrante; además, los gobiernos buscan institucionalizar y separar espacialmente a la población, mientras que la población residente es objeto de incitación programática al racismo y a la xenofobia (Zaviršek, 2017). El eurocentrismo racista representa a Occidente como democrático y a los inmigrantes como no democráticos, no distingue entre individuos y regímenes políticos, no toma en consideración las situaciones de emergencia de las que se 
trata este fenómeno: es, pues, una forma de humanitarismo armado.

Foucault dijo que en el liberalismo la libertad no es un hecho, sino algo que se produce constantemente: "El liberalismo no es la aceptación de la libertad; se propone fabricarla constantemente, despertarla y producirla, con, por supuesto, [el sistema] de restricciones y los problemas de costo que plantea esta producción" (Foucault, 1988, p. 65). Con el fin de evitar que nuestro mundo siga ardiendo, los trabajadores sociales deben ponerse al frente de los que pueden, y deben hacer una diferencia (Zaviršek, 2017).

El proceso de cambio político que se está originando en muchas partes del mundo también puede tener repercusiones desestabilizadoras, provocando nuevos movimientos de personas. Todas estas tendencias significan que los gobiernos probablemente enfrentarán crisis recurrentes que estimulan la migración y se acompañan de nuevas necesidades humanitarias; aunque gran parte de esta crisis de migración se llevará a cabo dentro de los países que se enfrentan a emergencias ambientales, también es probable que se produzcan movimientos transfronterizos (por ejemplo, el caso de la migración de venezolanos a Colombia entre 2016 y 2017).

La llegada de cientos de miles de venezolanos a territorio colombiano en la última década se ha convertido en un fenómeno socioeconómico en Colombia. La compleja situación política por la que está atravesando Venezuela es la principal causa de una migración, que en su mayoría está compuesta por personas altamente calificadas y que han ido insertándose exitosamente en diversos sectores productivos.

Aunque para Colombia la llegada masiva de venezolanos sea un eslabón más de una historia común, el hecho de pasar de ser un país emisor de migración internacional para ahora también ser uno receptor, es un fenómeno nuevo. Según cifras de Migración Colombia, en el 2012 entraron al país 262 mil venezolanos, 40 mil más que en el 2011 y 205 mil más que en 1999. Es una población móvil que frecuentemente entra y sale del territorio, pero que sin lugar a dudas está configurada una comunidad migrante, de la cual se cree que 28000 residen en Bogotá D. C., otros se ubican en ciudades más próximas a la frontera.

Es evidente que vivimos en un mundo interdependiente, y siendo Venezuela el país con el que más nexos e historia se comparte, lo que suceda en su interior trasciende la frontera. Por otro lado, las migraciones son la presentación real entre las sociedades, acercan, vinculan y presentan desafíos en todos los frentes (sociales, culturales, económicos y ambientales), desafíos que hacen más complejas las relaciones entre los dos Estados, pero a la vez las enriquecen y las llenan de oportunidades

\section{Las migraciones humanas como causa y consecuencia de las crisis ambientales}

Martin, Weerasinghe y Taylor (2013), aseguran que las crisis humanitarias, definidas como situaciones en las que existe una amenaza generalizada para la vida, la seguridad física, la salud o la subsistencia básica que está más allá de la capacidad de afrontamiento de las personas y las comunidades en las que residen, suelen tener implicaciones significativas para la migración. De acuerdo con estos autores, entre los recientes ejemplos se destacan el desplazamiento de millones de personas afectadas por el conflicto en Siria, el éxodo de personas desnutridas por la hambruna de Somalia y las evacuaciones tras el triple desastre de Japón.

Las crisis humanitarias pueden ser el resultado de eventos agudos, como los peligros naturales y humanos, los terremotos, los ciclones, los accidentes nucleares y los conflictos o los procesos de lento comienzo que causan daños ambientales, erosionan los medios de vida y, en casos extremos, conllevan a la hambruna al presentarse un agotamiento irreversible de los recursos naturales. Estos eventos y procesos pueden ser el desencadenante del movimiento, pero en la mayoría de los casos, los factores estructurales subyacentes como la pobreza y la mala gobernanza, constituyen el contexto explicativo de las migraciones masivas.

Entre 2010 y 2012 se registraron alrededor de 700 desastres naturales en todo el mundo, con más de 450 millones de personas afectadas (Martin et al., 2013). El conflicto armado o la inestabilidad política en Irak, Siria, Pakistán, Libia, Malí, Côte d'Ivoire, Sudán del Sur y la República Democrática del Congo, aportaron algunos millones más. Los conflictos prolongados en países como Somalia y Afganistán han producido movimientos irregulares secundarios, resaltando los complejos y exigentes problemas creados en situaciones de desplazamiento inducido por el conflicto armado.

El triple desastre en Japón dejó 19000 muertos o desaparecidos y dejó sin hogar a unas 400000 personas que también migraron a otros lugares, generando una carga adicional (pero involuntaria) sobre los escasos recursos naturales y medios de vida locales preexistentes.

Es claro que el fenómeno migratorio que es causa y consecuencia de problemas económicos y sociales también tiene orígenes ambientales, el cambio climático, la variabilidad climática, los eventos ambientales extremos, la perdida irreversible de activos naturales como la calidad de la tierra y la biodiversidad que sustenta son algunos de ellos. Los acontecimientos que pueden desencadenar crisis agudas e inducir desplazamientos incluyen: peligros naturales extremos tales como tormentas tropicales severas (huracanes Mitch y Stan en Centroamérica, ciclón Nargis en Birmania / Myanmar, y huracán Katrina en los Estados Unidos); tsunamis (por ejemplo, Indonesia, 
Sri Lanka y Japón en 2004); inundaciones por eventos climáticos extremos (por ejemplo, Pakistán y Colombia en 2010); terremotos (por ejemplo, Haití en 2010).

Martin et al. (2013), señalan que la falta de preparación para emergencias - como estándares de construcción adecuados, por ejemplo-, los altos niveles de pobreza y las debilidades en las capacidades locales y nacionales, se combinan para precipitar, y a veces perpetuar, las crisis humanitarias. Por ejemplo, en 2010, un terremoto de magnitud 7.0 en Haití provoco la muerte de más de 200 000 personas y desplazó a más de dos millones, pero unos meses después un terremoto mucho más severo en Chile (8.8 de magnitud) provocó unas 523 muertes. Los países muy ricos tampoco son inmunes, se vio durante el huracán Katrina en los Estados Unidos, paro los países estables y económicamente avanzados generalmente tienen mayor capacidad para asistir a las poblaciones afectadas.

La geografía constituye otra dimensión en este análisis. Durante la Guerra Fría, el principal foco de atención de la comunidad internacional era proteger a los desplazados a través de las fronteras internacionales (Martin, 2010), muchos de los tratados como refugiados habían sido expulsados de los países comunistas o habían sido desplazados por tropas de poder. El final de la Guerra Fría $\mathrm{y}$ las intervenciones posteriores aumentaron la visibilidad de las personas desplazadas por la fuerza dentro de las fronteras.

En la actualidad, el desplazamiento interno supera los movimientos transfronterizos de refugiados (Global Trends, 2013). La mayoría de los desplazamientos transfronterizos ocurren en países vecinos, los cuales tienen recursos financieros limitados. Jordania y Líbano, países azotados por la inestabilidad regional, los limitados recursos naturales y la guerra civil en el caso del Líbano, siguen recibiendo millones de personas de Siria.

La mayoría de los movimientos desencadenados por los impactos ambientales tienden también a ser internos o transfronterizos en los países vecinos (Martin et al., 2013), esta migración puede plantear desafíos a las comunidades y países de acogida, ya que las partes receptoras probablemente tendrán pocos recursos y pueden carecer de las estructuras legales o capacidad institucional necesarias para responder a las necesidades de los migrantes en crisis.

La proximidad geográfica puede también significar que las áreas de destino enfrentan algunos de los mismos retos ambientales que las áreas de origen migratorio, como la sequía y la desertificación, por lo tanto, pueden tener poco que ofertar, además de una mayor degradación ambiental y de su biodiversidad. En el peor de los casos, el movimiento hacia estas áreas puede intensificar la competencia y agotar los escasos recursos, profundizando las ya existentes crisis socioambientales. Ejemplos de ello son los brotes recurrentes de violencia entre refugiados y comunidades de acogida cerca del campamento de Kakuma en Kenia, y las tensiones entre un creciente número de pastores de Darfur y las comunidades locales en el este de Chad, debido a la rivalidad sobre comida y agua, para ellos y su ganado.

Una dimensión final desde la visión ambiental se relaciona con las necesidades de las poblaciones afectadas, es decir, su nivel de vulnerabilidad y su resiliencia; en gran medida, la vulnerabilidad es una medida de factores demográficos y socioeconómicos, algunos grupos son inherentemente vulnerables durante las crisis humanitarias como niños pequeños, ancianos y discapacitados, por nombrar algunos. Cuando son desplazados, estos grupos requieren una atención especial para asegurar que sus necesidades especiales de asistencia y protección sean atendidas. Otros pueden volverse vulnerables porque pierden sus sistemas de apoyo social o económico en períodos de crisis y desplazamiento, ejemplos de estos son las jefas de hogar, las personas de extrema pobreza y las víctimas de la trata de personas.

Las crisis con implicaciones migratorias probablemente no desaparecerán en el futuro, de hecho, el número y la frecuencia de estas crisis pueden aumentar sustancialmente en los próximos años. Se prevé que el cambio climático generará desplazamientos internos e internacionales sustanciales debido al aumento de la intensidad y la frecuencia de los peligros naturales, al aumento del nivel del mar, a la sequía persistente y a la desertificación y, potencialmente, a nuevos conflictos sobre los recursos escasos (Martin et al., 2013).

Dun y Gemenne (2017) plantean que en una visión "verdaderamente transversal" del fenómeno migratorio del siglo XX y XXI, la relación entre la crisis ambiental y las migraciones, debe ser considerada con mayor profundidad, no solo desde el análisis académico, sino desde el accionar político, plantean que, en primera instancia, es fundamental establecer una definición o conceptualización de la migración por motivos medioambientales e incluirla en el desarrollo de políticas de respuesta para tratar esos flujos, que no son consecuencia directa de una situación de violencia o carencia económica, sino que son el bucle de un sistema que se retroalimenta a sí mismo. No obstante, dos factores esenciales que revelan la necesidad de una definición pueden obstaculizar su elaboración:

- En primer lugar, muchos académicos quisieran establecer la migración por motivos medioambientales como un ámbito específico dentro de los estudios sobre migración (Avendaño y Aguilar, 2014). Existe una tendencia a aislar este campo y a considerarlo independiente de las teorías clásicas sobre migración (como si la migración por factores medioambientales fuera de otro tipo), sería más ventajoso intentar integrar los factores medioambientales en los estudios existentes sobre la migración, de esta forma sí contribuiría a una 
visión realmente transversal del fenómeno y sus consecuencias humanitarias.

- En segundo lugar, hay un afán generalizado por parte de periodistas y responsables políticos por disponer de cifras y previsiones; así, para que su investigación sea pertinente desde el punto de vista de la política, muchos se sienten obligados a formular hipótesis sobre los que son o pueden llegar a ser "desplazados por motivos ambientales". Evidentemente, estas cifras han de basarse en una de noción clara sobre quién se considera migrante ambiental, pues existe una tendencia a ampliar la noción para abarcar a cuantas personas sea posible; no obstante, una noción de migración por motivos ambientales demasiado amplia perjudicaría a los que necesitan más protección (Dun y Gemenne, 2017).

De acuerdo a la investigación de Avendaño y Aguilar (2014), el tema de los desplazados o refugiados ambientales se ha convertido en una situación de emergencia global, las poblaciones que a causa de los problemas del medio ambiente son trasladadas o reubicadas en otras ciudades o regiones distintas a las de su residencia son denominadas desplazados ambientales. Desastres, como terremotos, huracanes o tsunamis que causan devastación, generan desertificación, inundaciones, sequías y cambio climático, así como los accidentes industriales, están directamente relacionados con problemas de escasez de agua, energía, alimentos, biodiversidad y contagio de enfermedades, lo que induce a las poblaciones a desplazarse a otros lugares con pocas o ninguna posibilidad de retorno a sus tierras.

$\mathrm{Al}$ hacer referencia de los desplazados ambientales, cabe resaltar que estos no solo se trasladan a zonas aledañas dentro del mismo país, también cruzan áreas fronterizas de otras regiones para reubicarse en otros países. El Alto Comisionado de las Naciones Unidas para los Refugiados (ACNUR, 2006) describe que en estos movimientos de población, miles de personas pierden la vida tras las rutas migratorias, debido a las políticas restrictivas de los países o regiones a las que se desplazan y, entre otras causas, por las barreras militares de estas zonas.

Según lo describe Myers (1997) la problemática se extiende también, y en mayor rango, hacia la población vulnerable, es decir, mujeres, niños y ancianos, con lo que estima que para los próximos años, no muy lejanos a la actualidad, el número de desplazados aumentará a 50 millones, cifra de refugiados mayor que las que se generaron durante los grandes conflictos bélicos.

La base primordial de sustento para toda clase de recursos que mantiene a las poblaciones es el medio ambiente, por tanto, el deterioro y la degradación de este es la causa más relevante del desplazamiento de las poblaciones, ya sea a nivel interno o traspasando fronteras hacia otras regiones con el fin de asegurar su subsistencia.
Los desastres, tanto de ámbito natural ocurridos a causa del cambio climático junto con las consecuencias que este origina (erosión de los suelos, desertificación, deslizamientos, terremotos, inundaciones, entre otros), como en un ámbito antropogénico (accidentes industriales, conflictos bélicos, etc.), dan como resultado la migración y asentamiento de personas en lugares distintos a los de su origen (Avendaño y Aguilar, 2014).

El objetivo de disminuir el número de personas en esta condición es clave, pues con esto también se disminuirían algunas formas de empobrecimiento como el desempleo, el aumento de personas sin vivienda, la marginación económica, el incremento de la morbilidad y la escasez de alimento, evitando, en cierta medida, la desintegración social.

Finalmente, los desplazados ambientales son personas que también se encuentran respaldadas por los derechos humanos internacionales, en esta posición merecen que se les reconozca dejando de lado las amenazas a su integridad y supervivencia, otorgándoles principalmente derecho de asilo y resguardo.

Palma (2015), destaca que la migración humana desde, a través de y hacia Colombia ha sido poco explorada en los estudios internacionales, también es escaso el desarrollo de un cuerpo teórico multidisciplinar para entender las oportunidades y los desafíos que pueden surgir de este fenómeno. Al tiempo, la necesidad de generar políticas públicas integrales (sociales, económicas y ambientales) al respecto es creciente.

Palma (2015) cierra su disertación proponiendo cinco posibles áreas de intervención, frente a la transición de Colombia como país de emigrantes a inmigrantes:

- La primera, se refiere a la gestión de las remesas con políticas que contemplen la dimensión cultural del asunto.

- La segunda, se dirige a sacar provecho de las oportunidades que surgen de la pertenencia colombiana a la Alianza del Pacífico.

- La tercera, se perfila en pro de la reestructuración de los programas dirigidos a la repatriación de colombianos altamente calificados en el exterior.

- La cuarta, pone de relieve el acercamiento y entendimiento de la ventaja de nuestro país en temas como la mega diversidad y la riqueza en recursos naturales, elementos que se configuran en ventajas competitivas y en recursos de poder en el contexto internacional.

- La quinta, busca maximizar la gestión de Colombia como país de tránsito.

Estos deberían ser los temas de mayor atención, frente una agenda futura "realmente transversal" de la transición del fenómeno migratorio en un país como Colombia. Cabe destacar que esta sección del presente manuscrito se ha concentrado en los "migrantes ambientales". 
Colombia es un país altamente diverso, pluriétnico y multicultural, con grandes ventajas comparativas, gracias a su inmensa diversidad natural y climática, pero a la vez es un territorio vulnerable desde lo social y lo ambiental.

Mitigar, controlar y gestionar los flujos migratorios, no es un reto solo de las disciplinas del derecho internacional, la economía o el quehacer de los organismos gubernamentales responsables de los temas migratorios, es un reto "transversal" que involucra el reconocimiento y potenciación de los valores ambientales y culturales.

Además, en este contexto, es fundamental reconocer las vulnerabilidades (en especial al cambio y a la variabilidad climática), sin este reconocimiento explícito en las políticas y prácticas de ordenación ambiental del territorio, se terminará siendo un ejemplo más de una nación en colapso, ya no por el conflicto armado interno, sino por el mal manejo de los recursos naturales y por las crisis humanitarias que se desprenden de fenómenos migratorios causados por el deterioro ambiental y de la base fundamental de la vida en la tierra, que es su diversidad biológica.

\section{Referencias}

Alemán, F. (Agosto, 2017). Migraciones y acceso al trabajo en España. Trabajo presentado en Migraciones y su Transversalidad. VIII Congreso internacional del Programa de Derecho, Bogotá.

Alto Comisionado de las Naciones Unidas para los Refugiados (ACNUR). (2006). La situación de los refugiados en el mundo. Desplazamientos humanos en el nuevo milenio. Barcelona: Icaria.

Avendaño, W. y Aguilar, D. (2014). Geopolítica y medio ambiente: una mirada a la problemática de los desplazados ambientales. Investigación y Desarrollo, 22(2), 283-308.

Bernik, T. (Junio 11 de 2016). Španci: ¡Turisti, pojdite domov!. Dnevnik Daily. Recuperado de https://www.dnevnik.si/1042740751

Bradol, J. (2004). A critical international order and humanitarian action. In F. Weissman. (Ed.), In the Shadow of 'Just Wars' Violence, Politics and Humanitarian Action (pp. 1-24). London: Hurst and Company
Dun, O. y Gemenne F. (2017). Definir la migración por motivos medioambientales. Cambio Climático y Desplazamiento, 31, 10-11.

Foucault, M. (1988). E Birth of Biopolitics. Lectures at the College de France 1978-1979. London: Palgrave, Macmillan.

Global Trends. (2013). United Nations High Commissioner Refugees. Recuperado de http://www.unhcr.org/statistics/country/5399a14f9/unhcr-global-trends-2013.html

Holland, T., Peterson, G. y González, A. (2009). A Cross-National Analysis of How Economic Inequality Predicts Biodiversity Loss. Conservation Biology, 23(5), 1304-1313.

The Guardian. Hungarian prime minister says migrants are 'poison' and 'not needed'. (Julio 27 de 2010). The Guardian. Recuperad de https://www.theguardian.com/world/2016/jul/26/hungarian-prime-minister-viktor-orban-praises-donald-trump

Martin, S. (2010). Rethinking the International Refugee Regime in Light of Human Rights and the Global Common Good. En Hollenbach, D., Driven From Home: Protecting the Rights of Forced Migrants. (pp. 15-33). Washington, D. C.: Georgetown University Press.

Martin, S., Weerasinghe, S. y Taylor, A. (2013). Crisis Migration. Brown Journal of World A airs,1, 123-137.

Myers, N. (1997). Environmental refugees. Population and Environment, 19(2), 167-182.

Palacios, M. (2017). Los retos de Colombia en materia migratoria. Trabajo presentado en Migraciones y su Transversalidad. VIII Congreso internacional del Programa de Derecho, Bogotá

Palma, M. (2015). ¿País de emigración, inmigración, tránsito y retorno? La formación de un sistema de migración colombiano. Oasis, 21, 7-28.

Rockström J. et al. (2009). A safe operating space for humanity. $\mathrm{Na}$ ture, 461, 472-475.

Zaviršek, D. (2017) The humanitarian crisis of migration versus the crisis of humanitarianism: current dimensions and challenges for social work practice. Social Work Education, 36(3), 231-244. 